\title{
Christelle Avril, Les aides à domicile. Un autre monde
} populaire

Paris, La Dispute, coll. « Corps, santé, société », 2014

\section{Nehara Feldman}

\section{(2) OpenEdition}

\section{Journals}

\section{Édition électronique}

URL : http://journals.openedition.org/travailemploi/6931

DOI : 10.4000/travailemploi.6931

ISSN : 1775-416X

Éditeur

DARES - Ministère du Travail

\section{Édition imprimée}

Date de publication : 1 janvier 2016

Pagination : 197-199

ISSN : 0224-4365

\section{Référence électronique}

Nehara Feldman, «Christelle Avril, Les aides à domicile. Un autre monde populaire », Travail et Emploi [En ligne], 145 | janvier-mars 2016, mis en ligne le 01 janvier 2016, consulté le 23 septembre 2020. URL http://journals.openedition.org/travailemploi/6931; DOI : https://doi.org/10.4000/travailemploi.6931 


\title{
Notes DE LECTURE
}

\section{Les aides à domicile. Un autre monde populaire}

\author{
Christelle Avril
}

Paris, La Dispute, coll. « Corps, santé, société », 2014, 292 p.

\section{Lu par Nehara Feldman}

Comme son titre l'indique, l'ouvrage nous plonge dans le monde des aides à domicile, monde jusque-là délaissé aussi bien par les politiques que par les sociologues. L'auteure nous fait ainsi découvrir le quotidien de femmes appartenant au salariat non qualifié et dont la majeure partie de l'activité se déroule aux domiciles de personnes âgées auprès desquelles elles accomplissent trois ensembles de tâches : celles touchant directement leur corps (toilette et habillage) ; celles consistant à satisfaire leurs demandes (préparation des repas et courses notamment) ; celles concernant, enfin, l'entretien du logement. Qualifié par l'auteure de « relationnel », leur travail implique un effort spécifique dû à l'intervention sur et auprès de personnes atteintes par les effets indésirables du vieillissement. Situées au bas de l'échelle des intervenants professionnels à domicile, ces travailleuses sont régulièrement confrontées à des pénibilités physiques et psychiques, ainsi qu'à des contraintes temporelles.

Christelle Avril nous expose donc la condition d'aide à domicile qui semble a priori se trouver « aux antipodes du travail ouvrier» (p. 133). Mais elle ne se limite pas à traiter des conditions de travail, des pratiques professionnelles et du sens que les aides à domicile leur attribuent. Elle explore aussi l'ensemble de l'environnement social. Ainsi, ses analyses dépassent les questions relevant uniquement du domaine de la sociologie du travail : à travers le prisme du genre et celui des relations interraciales, l'auteure interroge la dimension inter-sectionnelle des dynamiques sociales observées, résultant du croisement de ces différents rapports de pouvoir. Cette démarche lui permet de mettre en lumière les lignes de clivage et les tensions au sein du milieu étudié. À un niveau plus général, l'ouvrage se présente donc comme une contribution à l'étude des milieux populaires en France, en examinant la place qu'y occupent les aides à domicile et en s'interrogeant sur « ce qu'elles ont, ou pas, en commun avec les pôles populaires déjà éclairés par les recherches sociologiques mais aussi quel rôle elles-mêmes jouent, ou pas, dans l'identité collective de ces milieux » (p. 17).

\footnotetext{
* Centre universitaire de recherche sur l'action publique et le politique - Épistémologie et sciences sociales (Curapp-ESS), université de Picardie Jules Verne.
} 
Pour atteindre ces objectifs, l'auteure mobilise une très riche enquête ethnographique comportant une étude exploratoire de deux ans, durant lesquels elle a elle-même travaillé comme aide à domicile, suivie par une enquête de terrain de trois ans au sein d'une association gérant le travail d'environ soixante-quinze aides à domicile. Dans le même temps, l'auteure a consulté les archives de la structure étudiée et dépouillé des revues spécialisées sur ce secteur d'activité. Une analyse statistique basée sur l'exploitation de l'enquête Emploi de 2003 et de 2011, de l'enquête Conditions de travail de 2005 et du Recensement de la population de 2007 ainsi qu'une étude sociohistorique du métier complètent les informations recueillies. L'ensemble du matériau est analysé subtilement grâce à la mobilisation des outils théoriques relevant de différents champs de la sociologie (travail, classes populaires, genre, relations interraciales/interethniques).

La première partie de l'ouvrage, intitulée « Travail, position, positionnements », présente les conditions concrètes du travail des aides à domicile. Elle décrit la manière dont s'est déroulée l'enquête de terrain et expose les données qui ont servi pour l'analyse statistique, puis revient sur l'histoire et les représentations sociales du métier. L'auteure détaille alors la position des aides à domicile dans l'espace social en interrogeant plus particulièrement leur inscription au sein des classes populaires. Elle explore enfin, et surtout, le positionnement des aides à domicile face à leur travail. Dans ce chapitre, C. Avril livre deux conceptions divergentes du travail, qui structurent le monde des aides à domiciles et font l'objet d'une analyse approfondie dans les deuxième et troisième parties de l'ouvrage : une partie d'entre elles est « tournée vers un certain pôle des classes populaires » alors que d'autres le sont « vers un certain pôle des classes moyennes-supérieures ».

La deuxième partie est ainsi consacrée aux aides à domicile issues de familles stables des classes populaires, installées de longue date dans la localité où se situe l'association (raison pour laquelle l'auteure les désigne comme des « déclassées autochtones »). Dans un premier temps, l'auteure expose la conception qu'ont ces aides à domicile de leur travail et démontre qu'elle s'inscrit « dans le prolongement et le maintien des relations qu'elles entretiennent avec leur milieu social d'origine » (p. 144). Elle examine ensuite comment cette fraction des aides à domicile se consolide en tant que "nous au féminin ", "s'opposant à un double "elles", celui du "haut" (les femmes très diplômées) et celui du "bas" (celles qu'elles appellent les "Noires" et les "Arabes") » (p. 263). L'analyse de la manière dont ces femmes négocient leur position sociale à la fois dans leur univers professionnel et plus généralement au sein des classes populaires aboutit à une proposition de définition d'un style de féminité qui leur est propre.

Dans la troisième et dernière partie de l'ouvrage, C. Avril s'intéresse à la catégorie d'aides à domicile qu'elle décrit comme «tournées vers un certain pôle des classes moyennes-supérieures ». Elle concerne des aides à domicile réparties en deux sous-groupes correspondant à deux types de trajectoires distincts. L'auteure distingue ainsi celles qu'elle désigne comme « les déclassées mobiles ». Venant pour la plupart des départements et territoires d'outre-mer ou de l'étranger (Maghreb, Afrique noire 
francophone et Haïti), issues de familles ayant du capital économique ou culturel, ces femmes ne réussissent pas à faire reconnaître leurs diplômes en France métropolitaine. C'est à défaut d'autres options qu'elles se reconvertissent professionnellement dans l'aide à domicile aux personnes âgées. L'autre sous-catégorie réunit celles qualifiées de " promues » : elle regroupe aussi bien des femmes françaises nées de parents français en rupture avec leur milieu familial que des filles d'immigrés et des étrangères issues de milieux pauvres. Toutes appartiennent à la « strate prolétarienne » des classes populaires. Décrivant la conception qu'ont ces femmes du travail et les relations qu'elles tissent au sein de leur univers professionnel, l'auteure constate l'existence d'alliances avec les femmes diplômées de classes moyennes-supérieures qui les encadrent - affinité qui, selon l'auteure, n'est pas sans lien avec le style de féminité investi par les premières.

Dans la conclusion, l'auteure propose des pistes politiques concernant la prise en charge salariée des personnes âgées dépendantes.

Parmi les nombreux apports de l'ouvrage, citons tout d'abord celui de nous faire découvrir un monde. Grâce notamment à la mobilisation d'extraits du journal d'enquête qui donne l'impression de ne pas seulement lire la description d'une scène mais de l'observer, le lecteur/la lectrice approche de manière approfondie l'expérience sociale de ces aides à domicile. Ceci est évidemment lié à la qualité de l'écriture, mais également à la rigueur de l'enquête ethnographique et à la finesse de l'analyse. L'ouvrage est donc un outil précieux d'apprentissage de la méthode ethnographique : on peut par exemple en tirer des enseignements concrets à partir de la façon dont la chercheure investit son terrain d'enquête et dont elle négocie sa position sociale auprès de ses interlocutrices.

La qualité de l'administration de la preuve, par de larges extraits de terrain commentés au plus près, permet par ailleurs d'approfondir la question relative à la qualification de comportements comme «féminins », « masculins » ou « virils », et nous semble ainsi ouvrir sur de nouvelles interrogations que nous partageons dans ce qui suit. Comment évaluer le poids déterminant du genre dans une action ou une interaction ? Certaines manières d'agir des aides à domicile, analysées par l'auteure comme des expressions de la « virilité au féminin », ne peuvent-elles être simplement interprétées comme relevant de leur appartenance aux milieux populaires ? De même, « la nouvelle autonomie publique » (p. 207), que constate l'auteure dans le mode de vie des aides à domicile les plus vulnérables, constitue-t-elle une transgression des normes genrées ou se résume-t-elle à un style de vie résultant de leur position marginale ? Les réponses à ces questions ne vont pas de soi et renvoient à un questionnement beaucoup plus large : les catégories « féminin », « masculin », « viril » peuvent-elles être appliquées à certaines pratiques en soi ? Sont-elles tributaires du sens que leur donnent les principaux/ principales concerné.e.s ? Le groupe étudié ? La société dans son ensemble, dont l'observatrice elle-même fait aussi partie ? Existe-t-il un consensus sur ces catégorisations ? Au-delà des qualités qui viennent d'être évoquées, l'ouvrage se présente ainsi comme une invitation à débattre et à poursuivre les investigations sur la coproduction des différents rapports sociaux et des formes ordinaires de catégorisation. 